Phys. Rev. Lett. 95, 170603, (2005)

\title{
First Passage and Cooperativity of Queuing Kinetics
}

\author{
Maria R. D'Orsogna and Tom Chou \\ Dept. of Mathematics, UCLA, Los Angeles, CA 90095 and \\ Dept. of Biomathematics, UCLA, Los Angeles, CA 90095
}

(Dated: November 6, 2018)

\begin{abstract}
We model the kinetics of ligand-receptor systems, where multiple ligands may bind and unbind to the receptor, either randomly or in a specific order. Equilibrium occupation and first occurrence of complete filling of the receptor are determined and compared. At equilibrium, receptors that bind ligands sequentially are more likely to be saturated than those that bind in random order. Surprisingly however, for low cooperativity, the random process first reaches full occupancy faster than the sequential one. This is true except near a critical binding energy where a 'kinetic trap' arises and the random process dramatically slows down when the number of binding sites $N \geq 8$. These results demonstrate the subtle interplay between cooperativity and sequentiality for a wide class of kinetic phenomena, including chemical binding, nucleation, and assembly line strategies.
\end{abstract}

PACS numbers: 02.50.Ey, 05.20.Dd, 05.60.-k

Cooperativity plays a key role in determining the equilibrium properties of queuing systems such as ligandreceptor binding, nucleation, melting of $\alpha$-helices, coupled chemical reactions, and assembly lines. Biophysical examples include $\mathrm{O}_{2}$ or $\mathrm{CO}$ binding to hemoglobin and myoglobin 1, 2, 3] and binding during cell signaling and morphogenesis [4, 5]. For a local bulk ligand concentration, the associated receptors will typically have a fraction of sites filled. The kinetics of queuing in these processes can exhibit diverse and rich behavior. A receptor may need to have a critical number of bound ligands before it can signal the next biochemical step. Thus, it is important to know not only the equilibrium ligand occupancy, but also the mean time to first reach this critical occupancy, as a function of local ligand concentration and binding strength. Similarly, in nucleation processes such as $\alpha$-helix formation or melting, local helix turns can form randomly or sequentially. The first time a complete helix forms (or melts) will be an important ingredient in protein folding models [6]. First passage times also define extinction and fixation in birth-death processes [7, 8]. Equilibrium distributions and first passage times also arise in applications of queuing, where, for example, average computer loads and the first time that demand exceeds capacity should be distinguished [9].

In this Letter, we formulate and use a kinetic chain model to highlight subtleties of ligand adsorption and desorption, queuing, and cooperativity. Our model is presented in the language of ligand binding to a single receptor with $N$ active sites of which $0 \leq n \leq N$ are occupied by ligands at any given time. The order of the binding can be imposed in two limiting ways. As shown in Fig.1 the addition of each successive ligand can influence one other specific site and allow the next ligand to bind to, or unbind from, that site only (a case we will denote by the index $\alpha=0$ ). Alternatively, the allosteric effect (from e.g. a large scale conformational change) can

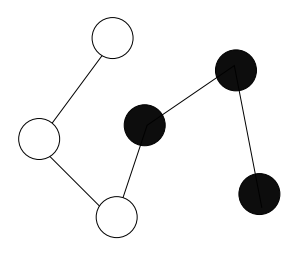

(a)

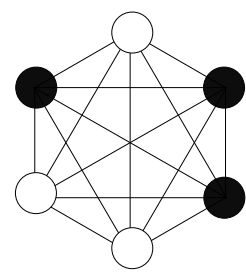

(b)
FIG. 1: Two limiting models of multiple ligand binding. In both cases, $N=6$, and $n=3$. (a) Sequential ligand binding/unbinding. At any given time, only one specific site can accept ligand adsorption and only one ligand can desorb. Upon defining the kinetics, this case will be denoted $\alpha=0$. (b) Random ligand binding/unbinding. An additional ligand can bind to any one of the open sites. Similarly, any one of the bound ligands can spontaneously desorb. This case will be denoted $\alpha=1$.

be spread equally to all remaining sites. The next ligand can bind to any one of these remaining open sites (a case we will denote by the index $\alpha=1$ ). Here, all bound ligand molecules are equally likely to spontaneously desorb. We do not consider mixed processes in which the binding order is sequential and the unbinding random, or vice versa. Thus, in our model, ligand binding occurs in a totally sequential manner as in Fig. 1h, or randomly as shown in Fig. 1b. We show how the binding order plays a crucial role in determining both equilibrium and kinetic properties: sequential ordering generally implies higher occupancy, while random ordering is associated with a shorter mean first passage to saturation. We also discover an intriguing regime of kinetic slowdown where different queuing rules result in dramatically different behaviors.

We define $P(n, t)$ as the probability that the receptor has $n$ bound ligands at time $t$, given that it had $n_{0}$ at 
time $t_{0}$. The evolution of the occupancy state $n$ can be mapped onto a one-dimensional random walk with a master equation given by $\partial_{t} P(n, t)=q_{n+1} P(n+1, t)-\left(q_{n}+\right.$ $\left.k_{n}\right) P(n, t)+k_{n-1} P(n-1, t)$. Here, $k_{n}$ and $q_{n}$ represent the ligand adsorption and desorption rates, respectively, when there are already $n$ ligands bound to the receptor. The probability density current from state $n+1$ to state $n$ is $J(n, t)=q_{n+1} P(n+1, t)-k_{n} P(n, t)$. The master equation, $\partial_{t} P(n, t)=J(n, t)-J(n-1, t)$, is solved under the constraint $\sum_{i=0}^{N} P_{i}=1$.

Different functional forms of $k_{n}$ and $q_{n}$ distinguish the sequential model from the random one. The number of ways for a ligand to bind an $n$ state receptor is proportional to the number of accessible binding sites. For sequential kinetics, at any given time, only one site is accessible for binding the next ligand, whereas for random binding, all $N-n$ empty sites are accessible. The number of accessible sites can thus be succinctly expressed as $(N-n)^{\alpha}$, where the index $\alpha=0$ corresponds to the sequential model, and $\alpha=1$ corresponds to the random one. Similarly, the number ways to detach a particle from an occupation state $n+1$ is $(n+1)^{\alpha}$. If conformational changes of the receptor molecule reach local thermodynamic equilibrium within the time scales required for ligand binding and unbinding, the rates obey detailed balance:

$$
\frac{k_{n}}{q_{n+1}}=z\left(\frac{N-n}{n+1}\right)^{\alpha} \exp \left[-\Delta G_{n}\right]
$$

The proportionality constant $z \approx v[L]$ is dimensionless and depends on the bulk ligand concentration $[L]$ and on the capture volume per binding site $v$. Typical values of $[L]=1 \mu M$ and $v=1 \mathrm{~nm}^{3}$ give $z \approx 10^{-6}$. The quantity $-\Delta G_{n} \equiv G_{n}-G_{n+1}$ is the free energy change, in units of thermal energy $k_{B} T$, upon detachment of one ligand from state $n+1$ to state $n$. Equivalently, $\Delta G_{n}$ can be interpreted as the free energy change due to ligand addition from state $n$ to state $n+1$. Cooperativity can be defined as the additional proclivity for the $(n+1)^{s t}$ ligand to bind as $n$ increases. A simple model for the free energy of binding is $\Delta G_{n}=-\varepsilon_{0}(n+1)^{\gamma}$ where $\varepsilon_{0}$ is the nonnegative ligand binding energy in units of $k_{B} T$. The nonnegative parameter $\gamma$ controls the cooperativity of successive binding. For $\gamma=0, \Delta G_{n}=-\varepsilon_{0}$ is independent of $n$ and the binding is noncooperative. If $\gamma>0, \Delta G_{n}$ decreases with $n$, hence a positive cooperative effect. Large values of $\gamma$ represent strong cooperativity. The equilibrium probability distribution $P_{e q}(n)$ is derived by imposing $J(n, t)=0$ and by using reflecting boundary conditions for the empty and full states, $k_{-1}, q_{0}=0$ and $q_{N+1}, k_{N}=0$, respectively [10]. Upon using Eq.[1]
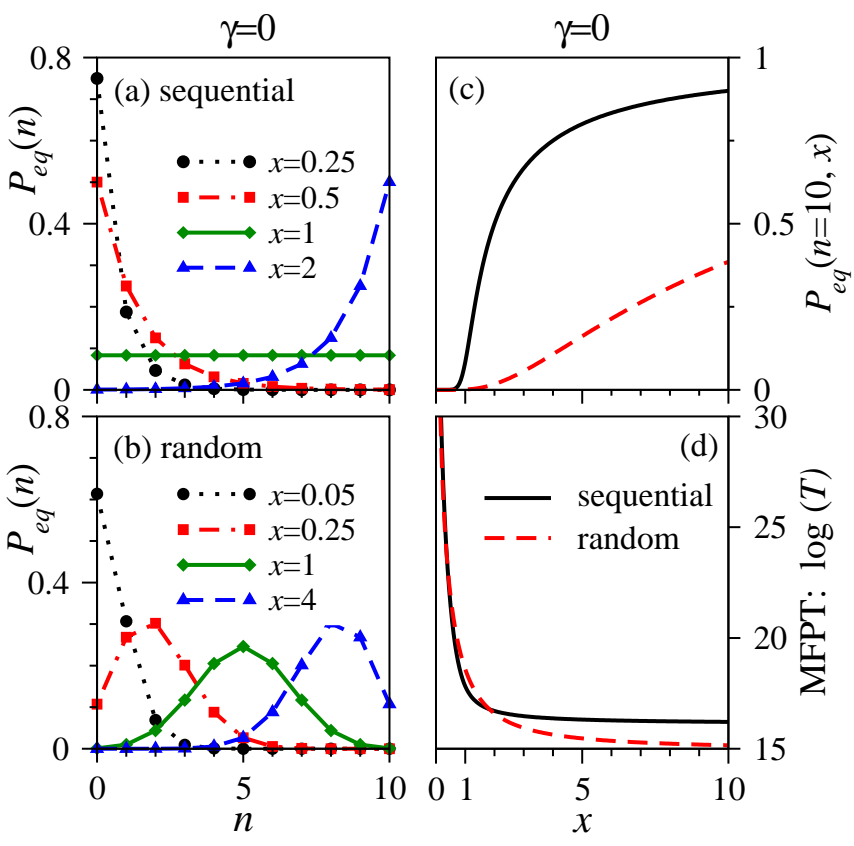

FIG. 2: Equilibrium occupation probabilities $P_{e q}(n)$ for $N=$ 10 and $\gamma=0$ as a function of ligand loading $n$ and various affinities $x$. (a) Sequential binding. (b) Random binding. Only the random model exhibits a probability maximum at intermediate $n$. This results from enhanced opposing drifts at small and large occupancies. The distributions for $\gamma \geq 1$ are qualitatively similar to those in (a). (c) The equilibrium probability of full occupancy $(n=N=10)$ for both models and $\gamma=0$ as a function of $x$. Full occupancy is more likely for the sequential case than for the random one. (d) The sequential and random case MFPT at $N=10$ and $\gamma=0$. Note the logarithmic scale.

$$
P_{e q}(n)=\frac{z^{n}\left(\begin{array}{c}
N \\
n
\end{array}\right)^{\alpha} \prod_{s=1}^{n} \exp \left[\epsilon_{0} s^{\gamma}\right]\left(1-\delta_{n, 0}\right)+\delta_{n, 0}}{1+\sum_{m=1}^{N} z^{m}\left(\begin{array}{c}
N \\
m
\end{array}\right)^{\alpha} \prod_{s=1}^{m} \exp \left[\epsilon_{0} s^{\gamma}\right]}
$$

For the noncooperative case, the occupation probabilities can be expressed in terms of a binding affinity $x \equiv z e^{\varepsilon_{0}}$. In Figs.2a-b we show the noncooperative $P_{e q}(n)$ for $N=10$ and various $x$. Only the random case allows an intermediate maximum of $P_{e q}(n)$ to develop as $x$ is increased. From Eq. [1 it can be seen that for small $n$ values the ratio $k_{n} / q_{n+1}$ is larger for the random case than for the sequential one. Conversely, the same ratio is smaller for large $n$. The random process thus induces an effective drift towards intermediate $n$ values. This is schematically shown in Fig. 3. We can rewrite Eq.1 in the form $k_{n} / q_{n+1}=\exp \left(-\Delta \bar{G}_{n}\right)$, where

$$
\Delta \bar{G}_{n}=\Delta G_{n}-\alpha \ln \left(\frac{N-n}{n+1}\right)-\ln z
$$




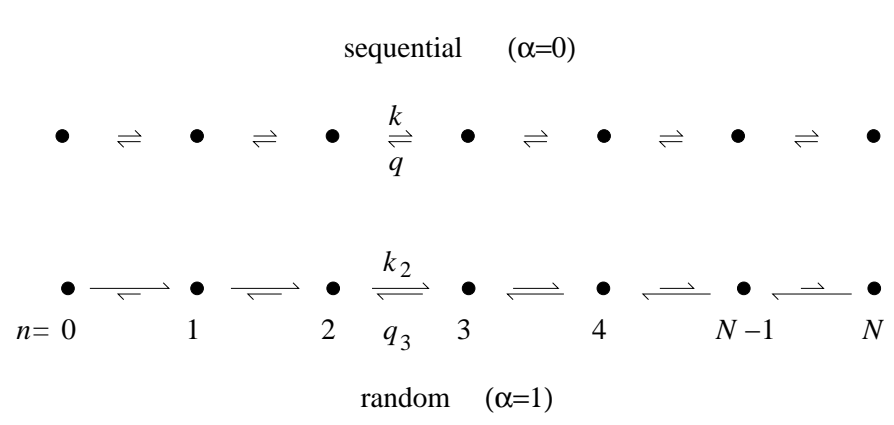

FIG. 3: Kinetic steps for $x \sim 1$ and $\gamma=0$. (a) Sequential process. All successive steps incrementing the number of bound receptors have the same rate $k$, while all decrement steps have the same rates $q$. (b) Random process. There are more ways to attach a ligand when $n$ is small. Similarly, there are more ways of removing receptors when there are many bound receptors. The effective rates push the system from both directions toward an intermediate occupation level. Given an initial condition at $n=0$, the first passage statistics to $n=N$ will be controlled more by the effective forward rates $k_{n}$ than the effective backward rates $q_{n}$. Therefore, the larger $k_{n}$ tending to load the receptor outweigh the effects of the larger $q_{n}$ tending for desorption. The overall MFPT is thus larger for in the random case than in the sequential one.

is the effective free energy change that includes the entropy associated with ligand binding and unbinding. Unlike $G_{n}$, the total effective free energy as a function of $n$,

$$
\bar{G}_{n}=G_{n}-\alpha \ln \left(\begin{array}{l}
N \\
n
\end{array}\right)-n \ln z,
$$

can have a minimum with respect to $n$ if $\alpha=1$. Thus, a maximum in $P_{e q}(n)$ can occur only in the random case. Equilibrium distributions and occupancy probabilities have been widely used in approximations of kinetics of biochemical reactions [10]. One commonly used metric is the filling fraction $f_{e q}(x ; \alpha, \gamma)=N^{-1} \sum_{n=1}^{N} n P_{e q}(n)$. We can compute $f_{\text {eq }}$ under either the sequential or random assumptions:

$$
\begin{aligned}
& f_{e q}(x ; 0,0)=\frac{x\left(1-(N+1) x^{N}+N x^{N+1}\right)}{N(x-1)^{2}+N x(x-1)\left(x^{N}-1\right)} \\
& f_{e q}(x ; 1,0)=\frac{x}{1+x},
\end{aligned}
$$

respectively. These two expressions coincide only in the infinite affinity limit $x \rightarrow \infty$, signifying that the process no longer depends on binding order. Expressions such as $f_{e q}$ have been used in kinetic equations describing binding to hemoglobin/myoglobin 1, 2], regulatory networks [11, viral infection dynamics [12], and cell signaling [13]. However, using equilibrium expressions in kinetic equations assumes separation of time scales or near steadystates. Moreover, filling fractions do not provide information about typical loading time scales. The queuing nonequilibrium properties are more telling, in this context, than their equilibrium counterparts and surprising effects may arise. In particular, faster complete occupation of a receptor can occur even though the mean occupancy is lower at equilibrium. The mean first passage time (MFPT) to a target filling can be evaluated by using standard techniques [14]. For a receptor in the unoccupied $n=0$ state at $t=0$ the MFPT to reach an $n^{*}$ filling is [15]

$$
T\left(n^{*} ; \alpha\right)=\sum_{n=0}^{n^{*}-1} \frac{1}{k_{n}}\left(1+\sum_{j=0}^{n-1} \prod_{i=j}^{n-1} \frac{q_{i+1}}{k_{i}}\right) .
$$

To evaluate Eq. (7) we need independent expressions for $k_{n}$ and $q_{n}$. We let $k_{n}=\nu v[L](N-n)^{\alpha}$ and $q_{n+1}=$ $\nu(n+1)^{\alpha} \exp \left[\Delta G_{n}\right]$, where $\nu$ is the frequency of reaction attempts. We choose $k_{n}$ to be independent of $\Delta G_{n}$ because for many systems, such as antibody-antigen complexes [16], cooperative effects are felt only in the detachment process. In these cases, the strength of a ligandreceptor chemical bond depends on the total number of bound ligands present. These existing ligands typically do not affect the kinetics of attachment. For the noncooperative case $(\gamma=0)$, the MFPT to full occupancy in units of $\nu^{-1}$ is [17]:

$$
T(N ; \alpha=0)=\frac{x}{z(x-1)}\left[\frac{x^{-N}-1}{x-1}+N\right] .
$$

Logarithms of the MFPT from $n=0$ to complete filling $n^{*}=N$ are plotted in Fig.21 for $\gamma=0$. A comparison with Fig.2 shows an unexpected effect. Although the noncooperative random process yields a lower equilibrium ligand population than the sequential process, its MFPT is shorter. This is due to an overall effective increase in the local diffusivity as evident from the form $k_{n} \propto(N-n)^{\alpha}$. However, near the half-filling affinity $x=1$, corresponding to $f_{e q}=1 / 2$, the MFPT of the random case is greater than that of the sequential one. This is emphasized in Fig. पh, where we plot the ratio of random to sequential MFPT as a function of binding energy. A striking peak develops for $x=1$ provided the receptor has at least $N_{c}=8$ binding sites. Indeed, as $N>N_{c}$ increases, the random MFPT increases dramatically. Why does this happen? From Fig.3 we see that for the random case near $x=1$, the backward rates $q_{n+1}$ balance the forward rates $k_{n}$. This results in a vanishing net drift at intermediate occupations. The effective potential $\bar{G}_{n}$ develops a minimum that for $x=1$ is exactly located at $N / 2$. The opposing drifts bias the filling to intermediate values forming a kinetic trap and delaying first saturation. The MFPT increases significantly due to the time required to escape this trap, despite the increase in effective diffusivity near the potential minimum. When $N \geq 8$, the trapping is strong enough that the MFPT of the random case is greater than that of the 
sequential process. This effect is disrupted as $x$ deviates from unity as the minimum in $\bar{G}_{n}$ disappears. Note that this behavior occurs only in the absence of cooperative effects: for $e . g ., \gamma=1$ the equilibrium occupancies follow the same trends as in Fig.2 and the random system
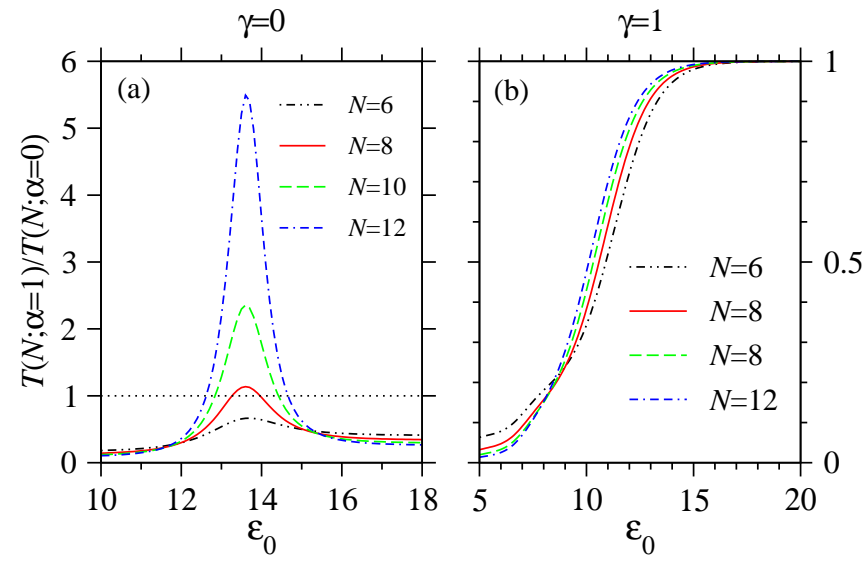

FIG. 4: Mean first passage time ratios $T(N ; 1) / T(N ; 0)$. (a) For $\gamma=0$, random binding $(\alpha=1)$ is faster except for a window of binding energies near $x=z e^{\varepsilon_{0}}=1$. For clarity we plot $T(N ; 1) / T(N ; 0)$ as a function of $\varepsilon_{0}$ for $z=10^{-6}$. The critical value $x=1$ occurs at $\varepsilon_{0}=13.8$. The peak scales as $\sim 2^{N} / N^{3}$. (b) For $\gamma=1$ (and in fact $\gamma \geq 1$ ), the random process always reaches full occupancy faster than the sequential one.

To explicitly see a size-dependent trap, consider the MFPT, $T_{\text {trap }}(M ; \alpha)$, out of a band of sites of length $2 M$, centered about $n=N / 2$. For $x=1, \gamma=0$, and an initial condition of $n=N / 2$, the logarithm of the ratio of the random $T_{\text {trap }}(M ; 1)$ to the sequential $T_{\text {trap }}(M ; 0)$ is plotted in Fig.5] The trapping effect is evident from the fact that for large enough $M$ the random $T_{\text {trap }}(M ; 1)$ can easily be $e^{10}$ times larger than the sequential $T_{\text {trap }}(M ; 0)$.

Our analysis reveals the conspicuous effects that cooperativity and sequence have on binding kinetics, especially on the MFPT to saturation. The key result is that equilibrium and dynamic measurements offer different answers as to whether the sequential or the random order is more efficient in ligand saturation. At equilibrium, sequential processes are more likely than random ones to saturate the ligand. For dynamic properties, such as MFPTs, random processes are faster. This is true except for large noncooperative systems $(N \geq 8)$ with a binding affinity near $x=1$, where random processes dramatically slow down. One can also consider the reverse process of emptying a completely filled receptor. The clearance time can be obtained by using particle-hole symmetry and the replacements $k_{p}^{*} / q_{p+1}^{*}=$ $[(N-p) /(p+1)]^{\alpha} \exp \left(\Delta G_{p}\right) / z$ where $p$ is the number of holes present.

The rate parameters used in our study can also be reinterpreted as the inverse of the mean servicing time

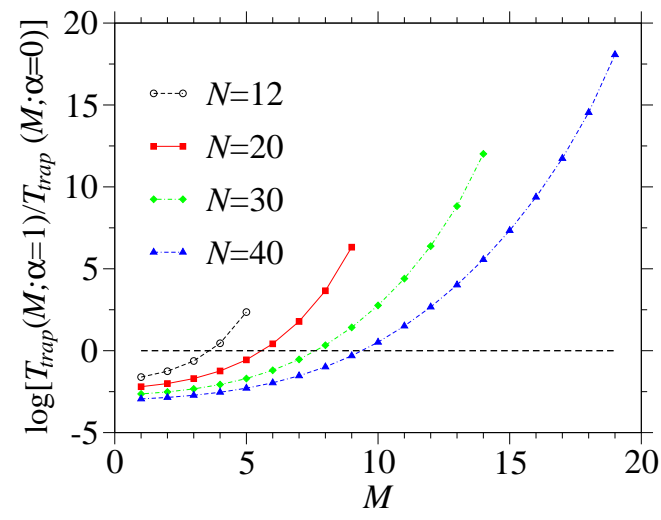

FIG. 5: Logarithm of the ratio $T_{\text {trap }}(M ; 1) / T_{\text {trap }}(M ; 0)$. Here, $x=1, \gamma=0$ and the initial occupancy is at $n=N / 2$. Negative values of the ratio imply that escaping a $2 M$ kinetic trap is faster for the random process than for the sequential one. This occurs only for small enough traps centered on $N / 2$ where the random dynamics is faster. For large enough values of trap length $2 M$ the effects described in Fig. [3 take place and the exit time of the random case, $T_{\text {trap }}(M ; 1)$, is much larger than the sequential one, $T_{\text {trap }}(M ; 0)$.

in a customer service queue. Our results indicate a rich set of outcomes depending on queue size, cooperativity, and sequentiality. Levels of "service discipline," or how to order customer service 9], can be implemented in different ways to achieve the desired outcome with highest probability, measured by average (equilibrium) or MFPT (nonequilibrium) attributes.

MRD thanks S. Vinogradov for helpful comments and the ARO for support through grant DAAD19-02-1-0055. TC acknowledges support from the NSF and NIH via grants DMS-0206733, DMS-0349195, and K25AI056872.

[1] C. J. Thompson, Mathematical Statistical Mechanics, (Macmillan, New York, 1972).

[2] R. E. Weber and S. N. Vinogradov, Physiol. Revs., 81, 569, (2001).

[3] N. Alberding, H. Fraunfelder, and P. Hänggi, PNAS, 75, 26, (1978).

[4] J. B. L. Bard, P. W. Barlow, and D. L. Kirk, eds., Morphogenesis: The Cellular and Molecular Processes of Developmental Anatomy, (Cambridge University Press, Cambridge, 1992).

[5] D. S. Burz, R. Riveria-Pomar, H. Jäckle, and S. Hanes, EMBO, 17, 5998, (1998); X. Ma, D. Yuan, K. Diepold, T. Scarborough, and J. Ma, Development, 122, 1195, (1996).

[6] D. Clarke, A. Doig, B. Stapley, and G. Jones, Biochemistry, 96, 7232, (1999).

[7] C. Doering, K. Sargsyan, and L. Sander, Multiscale Modeling and Sim., SIAM. 3, 283, (2005)

[8] S. Redner, A Guide to First Passage Processes, (Cambridge University Press, Cambridge, 2001).

[9] D. Gross and C. M. Harris, Fundamentals of Queueing 
Theory, 3rd ed., (Wiley, New York, 1998).

[10] N. G. Van Kampen Stochastic Processes in Physics and Chemistry (North Holland, Amsterdam, 1992, $2^{\text {nd }}$ ed)

[11] M. B. Elowitz and S. Leibler, Nature, 403, 335, (2000); T. S. Gardner, C. R. Cantor and J. J. Collins, Nature, 403, 339, (2000).

[12] P. W. Nelson, M. A. Gilchrist, D. Coombs, J. M. Hyman, and A. S. Perelson, Math. Biosci. Eng., 1, 267 (2004).

[13] N. J. Eungdamrong and R. Iyengar, Biol. of the Cell, 96, 355, (2004).
[14] C. W. Gardiner, Handbook of Stochastic Methods, (Springer, New York, 2004)

[15] P. A. Pury and M. O. Cáceres, J. Phys. A, 36, 2695, (2003).

[16] S. Kulin, R. Kishore, J. Hubbard and K. Helmerson, Biophys. J. 83, 1965 (2002).

[17] Note that Eqs.78 8 are derived in Ref. 15] as Eqs. (2.9), (6.2) respectively, with the substitutions $M, w_{-M}^{-} \rightarrow 0$, $\gamma \rightarrow x^{-1}$, and $L \rightarrow N-1$. 